\title{
Les peintures murales du Château Saint-Maire. Autour des modèles allégoriques du prince-évêque
}

\section{Karen Straub}

\section{(2) OpenEdition}

1 Journals

Édition électronique

URL : http://journals.openedition.org/edl/1369

DOI : $10.4000 /$ edl. 1369

ISSN : 2296-5084

Éditeur

Université de Lausanne

\section{Édition imprimée}

Date de publication : 1 décembre 2018

Pagination : 131-150

ISBN : 978-2-940331-69-7

ISSN : 0014-2026

\section{Référence électronique}

Karen Straub, «Les peintures murales du Château Saint-Maire. Autour des modèles allégoriques du prince-évêque », Études de lettres [En ligne], 3-4 | 2018, mis en ligne le 15 décembre 2020, consulté le 17 décembre 2020. URL : http://journals.openedition.org/edl/1369; DOI : https://doi.org/10.4000/edl. 1369

\section{(c) Études de lettres}




\section{LES PEINTURES MURALES DU CHÂTEAU SAINT-MAIRE. AUTOUR DES MODĖLES ALLÉGORIQUES DU PRINCE-ÉVÊQUE}

Dans le corridor de l'ancien palais épiscopal de Lausanne, on peut encore admirer l'ensemble de peintures murales allégoriques qui fut commandé par le prince-évêque Aymon de Montfalcon au début du XVI $\mathrm{I}^{\mathrm{e}}$ siècle. La source de ce décor peint est à chercher dans les poèmes allégoriques des Douze Dames de Rhétorique de George Chastelain et du Bréviaire des nobles d'Alain Chartier, transposés dans le média de la peinture monumentale. Des comparaisons avec les manuscrits enluminés éclairent la représentation voulue par l'évêque. Se posent aussi la question du choix des textes et celle, corollaire, de leur transmission; en y répondant, il nous sera possible d'avancer des hypothèses concernant les intentions présumées du commanditaire ${ }^{1}$.

Prince-évêque de Lausanne à partir de 1491, Aymon de Montfalcon (vers 1440-1517) fit effectuer des travaux de transformation de son palais épiscopal, le château Saint-Maire. Cet édifice du premier tiers du $\mathrm{XV}^{\mathrm{e}}$ siècle, situé tout au nord de la Cité, servait de résidence aux évêques et fut conçu au départ comme un bâtiment isolé et solidement fortifié 2 . Montfalcon modifia l'aspect du château par la construction d'un vestibule à l'ouest de manière à en faire une résidence plus représentative; à l'intérieur, il fit rénover au premier étage la "chambre de l'évêque» dont la grande cheminée et le plafond, avec sa devise et son monogramme,

I. Je remercie Jean-Claude Mühlethaler pour sa relecture attentive et constructive.

2. Voir M. Reymond, "Les châteaux épiscopaux, les hôtels de ville de Lausanne", p. 162; M. Grandjean, La ville de Lausanne, p. 345-361. Le château Saint-Maire remplaça l'ancienne résidence épiscopale située à côté de la cathédrale, aujourd'hui Musée historique de Lausanne. 
sont encore visibles. De surcroit, il commanda les peintures murales qui embellissent les parois des couloirs. Pour le corridor au rez-de-chaussée, Aymon choisit un ensemble sophistiqué de peintures allégoriques: les Douze Dames de Rhétorique sur la paroi nord, les Vertus des nobles sur la paroi sud (pl. IV). Vu leur état de préservation, l'analyse des peintures allégoriques rencontre quelques difficultés. Découvertes sous une couche de plâtre en 1908, elles étaient fortement endommagées, surtout dans la partie des figures qui fut complétée au cours d'une restauration après leur mise au jour ${ }^{3}$.

Les peintures couvrent l'ensemble des deux parois du corridor, soit $16 \mathrm{~m}$ sur environ $4 \mathrm{~m}$ de chaque côté ${ }^{4}$. La composition est divisée en plusieurs registres superposés: la zone en bas comporte des caissons octogonaux encadrés de pointes de diamant et suivis d'une frise d'acanthes. Au-dessus, la partie principale se subdivise sur chaque paroi en treize panneaux par des arcades de troncs d'arbre dont les branches forment un encadrement; sur la paroi nord, des colonnes les remplacent à deux reprises.

Chaque panneau présente une figure féminine à mi-corps tenant un grand rouleau de parchemin chargé de trois strophes qui occupe toute la longueur de l'arcade. La zone principale finit par les mots SI QVA FATA SINANT ("si les destins le permettent»), une citation empruntée à Virgile qui fut utilisée par Aymon de Montfalcon comme devise personnelle ${ }^{5}$, qui court tout le long du corridor. En haut est représentée une frise de rinceaux, de candélabres et de cornes d'abondance en alternance avec des centaures et des putti qui présentent des écussons.

3. Un graphique de la silhouette indique l'état fragmentaire et les parties conservées, consultable sur le site internet officiel <http://www.patrimoine.vd.ch/ accueil/chateau-saint-maire/le-chateau-actuel/description/le-decor-peint/renaissance/ la-fresque/les-fresques-description/>. De plus, des photographies en noir et blanc (conservées au Service des bâtiments, monuments, et archéologie de l'État de Vaud à Lausanne) montrent bien leur état avant et après la restauration. Pour la découverte et la restauration effectuée vraisemblablement par le peintre et restaurateur d'art Ernest Correvon (1873-1965), voir J.-A. Bohy, "Les peintures du château de Lausanne», p. 58; M. Grandjean, La ville de Lausanne, p. 368; L. Rochat, Les peintures du corridor du château Saint-Maire à Lausanne, p. 7-10. Je remercie Lise Rochat qui m’a fait parvenir un exemplaire de son mémoire de maîtrise.

4. M. Grandjean, La ville de Lausanne, p. 368.

5. La citation est tirée du premier livre de l'Énéide (I, 18); voir M. Grandjean, La ville de Lausanne, p. 368; K. Straub, "Se riens y a qui de noble umbrage" ", p. 281. 
D'autres frises d'acanthes et de rinceaux avec des écussons suivent audessus. Les frises se prolongent au-dessus de l'entrée (à l'ouest); deux figures supplémentaires avec des ailes tiennent un écusson aux armes du prince-évêque.

Les personnifications féminines adoptent des attitudes variées et sont habillées plus au moins selon la mode en vogue autour de $1500^{6}$. Leur représentation reste relativement générique et dans l'état actuel des peintures, seules cinq dames allégoriques se distinguent par des attributs. En conséquence, ce n'est qu'à l'aide du texte, soit des vers transcrits sur les murs, qu'on a réussi à identifier les personnifications de la paroi nord avec les Douze Dames de Rhétorique et celles de la paroi sud avec les Vertus des nobles?.

Quand on entre dans le corridor, le cycle des peintures murales commence par les Douze Dames. Les vers sont tirés d'une correspondance entre des poètes et dignitaires des cours de Bourbon et de Bourgogne. Jean Robertet, alors secrétaire du duc de Bourbon et poète, est à l'origine de l'échange épistolaire avec George Chastelain, fameux auteur et historiographe à la cour bourguignonne, soutenu par

6. Vu l'état fragmentaire, il est difficile de déterminer la nature de leurs habits. Ils semblent comparables aux vêtements que l'on retrouve dans les tableaux français et flamands vers la fin du XVe siècle et encore au XVI ${ }^{\mathrm{e}}$ siècle, comme sur les tapisseries Los Honores réalisées pour Charles V à Bruxelles et achevées en 1523. On peut retenir plus particulièrement l'exemple de la tapisserie représentant Prudentia: voir G. Delmarcel, Los Honores, p. 60 sq., pl. 2.1. La datation des peintures murales est encore une question ouverte. Les anciens auteurs considéraient l'un des graffitis sur les murs, qui indique « 1500 », comme une date authentique: voir J.-A. Bohy, «Les fresques pré-renaissantes du château de Lausanne», p. 139, 141 ; M. Grandjean, La ville de Lausanne, p. 368. F. Elsig propose une datation vers 1510 sur la base du style des frises de grotesques ( La peinture en Savoie et en Franche-Comté durant la première moitié du XVI e siècle", p. 85). L. Rochat suppose qu'elles ont été effectuées après le voyage de Montfalcon à Bruxelles en 1501, lors des négociations en vue du mariage du duc de Savoie avec Marguerite d'Autriche (Les peintures du corridor du château Saint-Maire à Lausanne, p. 73 et 78).

7. A. Piaget considère les personnifications du côté sud comme les Vertus des nobles ("Aymon de Montfaucon et sa cour littéraire», p. 465); il revient à M.-R. Jung d'avoir identifié les allégories moins connues des Douze Dames de Rhétorique ("Les "Douze Dames de Rhétorique”", p. 230 sq.). 
Jean de Montferrant, un noble au service de Philippe le Bon ${ }^{8}$. Le traité allégorique, avec les descriptions versifiées (les "Enseignes») des douze Dames, représente la partie centrale de cet échange de lettres dont la date est estimée des années 1462/63 9 . Le texte est connu, soit complet, soit en extraits, grâce à onze copies réalisées entre le $\mathrm{XV}^{\mathrm{e}}$ et le XVIII ${ }^{\mathrm{e}}$ siècle qui proviennent toutes des Pays-Bas bourguignons ${ }^{10}$. Quatre d'entre elles - dont trois en manuscrits indépendants - contiennent le texte intégral, y compris les illustrations représentant les suivantes de Dame Rhétorique. C'est le manuscrit (Cambridge, UL, ms. Nn. III.2) commandé par Jean de Montferrant, lui-même participant à l'échange épistolaire, qui a servi de modèle aux autres exemplaires illustrés. Dans ce manuscrit, réalisé à Bruges en 1467/68 et comportant quinze miniatures de style flamand, on trouve pour la première fois le cycle d'images «canonique» des Douze Dames de Rhétorique. Le manuscrit présente également à deux endroits les armes de la famille des Montferrand, ce qui a amené à identifier Jean comme étant le frère de l'évêque Benoît de Montferrand ${ }^{11}$.

La correspondance, ouverte au public aristocratique des deux cours, se présente comme un débat sur la création littéraire et le statut de l'auteur ${ }^{12}$. C'est à George Chastelain qu'on doit l'intervention allégorique des douze Dames dont les discours s'enchaînent pour formuler son idée de l'auteur idéal. Chacune des «compaignes de la Dame Rhétorique» ${ }^{13}$ indique quelles sont ses connaissances et définit ses caractéristiques de manière métaphorique. Il s'agit de: Science, Eloquence, Profundité,

8. Cf. George Chastelain, Jean Robertet, Jean de Montferrant, Les Douze Dames de Rhétorique, p. 10-20; K. Straub, "Les Douze Dames de Rhétorique" in Text und Bild, p. 17-24.

9. Pour la datation du texte, voir M.-R. Jung, «Les "Douze Dames de Rhétorique" ", p. 229 sq.

Io. À l'exception de l'exemplaire conservé à Rouen, Bibliothèque municipale, ms. 1234. Comme il a appartenu à Jean Derval (mort en 1482), on a supposé qu'il avait été exécuté en Anjou avant 1482; voir D. Cowling, in Les Douze Dames de Rhétorique, p. 36-59, qui donne une description de tous les manuscrits.

II. Les armes des Montferrand (l'orthographe du nom varie) se trouvent au fol. 9 et sur les fermoirs d'origine: cf. C. Chavannes-Mazel, "The Twelve Ladies of Rhetoric in Cambridge (CUL MS Nn.3.2)», p. 144 sq. et 144-147 pour la datation des manuscrits; K. Straub, "Les Douze Dames de Rhétorique” in Text und Bild, p. 228 et 237-240.

I2. J.-C. Mühlethaler "Un manifeste poétique de 1463 », p. 84; E. Doudet, "Poétique en mouvement», p. 83 sq.

13. Cité d'après George Chastelain, Jean Robertet, Jean de Montferrant, Les Douze Dames de Rhétorique, p. 126, v. 137. 
Gravité de Sens, Vieille Acquisition, Multiforme Richesse, Flourie Memoire, Noble Nature, Clere Invention, Precieuse Possession, Deduction Louable et Glorieuse Achevissance.

Dans les manuscrits enluminés, la description de chacune des douze Dames commence systématiquement par une miniature présentant la personnification qui est accompagnée par l'inscription de son nom et d'une sorte de devise, tirée la plupart du temps de la Vulgate ${ }^{14}$, ellesmêmes suivies de trois strophes en vers. Les Dames évoquent les conditions de la création littéraire en recourant à des métaphores dans un discours qui reste essentiellement abstrait. Seuls les noms de trois des dames - Eloquence, Flourie Memoire et Clere Invention - renvoient aux concepts de la rhétorique classique; les descriptions ne comportent guère de vocabulaire se rapportant directement à l'acte d'écrire ${ }^{15}$.

Les Douze Dames offrent une poétique qui peut être considérée comme représentative de l'idée que se font de leur métier les "(grands) rhétoriqueurs ${ }^{16}$ au tournant des $\mathrm{XV}^{\mathrm{e}}-\mathrm{XVI}{ }^{\mathrm{e}}$ siècles. Ceux-ci étaient à la fois historiographes et poètes, servant de secrétaires et de chroniqueurs aux princes français et bourguignons. Se considérant volontiers comme des "orateurs " ${ }^{17}$, ils revendiquaient une connaissance approfondie de la première rhétorique (la prose) comme de la seconde (la poésie). Conscients de leur dignité d'écrivain, ils se sentaient obligés de s'engager dans les domaines politique, moral et culturel par l'écriture ${ }^{18}$.

Leur idée clé d'une compétence à la fois poétique et éthique est au cœur des "Enseignes» des Douze Dames. Les strophes font ressortir l'image du poète parfait qui devait avoir des connaissances universelles et se distinguer par ses capacités morale et intellectuelle, toutes nécessaires à la création littéraire. L'image de l'auteur savant, de bonnes mœurs, inspiré par la sagesse divine et lui-même créateur ${ }^{19}$, se manifeste aussi dans les enluminures, étroitement liées au texte.

I4. Voir J.-C. Mühlethaler, «La citation éclairante».

I5. J.-C. Mühlethaler, «Un manifeste poétique de 1463 », p. 84.

16. Voir P. Zumthor, Le masque et la lumière, p. 9-22.

I7. Voir à ce sujet la contribution d'E. Doudet au présent volume.

I8. P. Jodogne, "Les "Rhétoriqueurs" et l'humanisme», p. 160 sq.

19. J.-C. Mühlethaler, "Un manifeste poétique de 1463 ", p. 99 sq.; E. Doudet, "Poétique en mouvement», p. 96. 
Cet ensemble élaboré, comment a-t-il pu être transposé dans un media monumental? En comparant le traité enluminé avec les peintures murales, on constate que le principe de présentation a été maintenu. Les peintures murales reprennent le schéma tripartite en image, citation transcrite sur une banderole et description de la personnification en vers.

Examinons à titre d'exemple la troisième dame, Profundité. Elle fait suite à Science et Eloquence, toutes les trois formant un groupe dans la mesure où elles prennent pour sujet le savoir universel donné de Dieu. Dame Science se pose en représentante de la philosophie et de la sagesse divine; Eloquence se rattache à la conception cicéronienne de la rhétorique en faisant ressortir la relation primordiale entre la sagesse et l'éloquence au service du public, tandis que Profundité représente un savoir encyclopédique ${ }^{20}$. Elle perçoit tout ce qui se trouve dans l'univers et dit d'elle-même que: "J'ay ciel, mer, terre en ma teste petite» ${ }^{21}$. Profundité va au fond des choses, car elle est chargée de les interpréter. Guidée par la philosophie et la théologie, elle peut se servir du savoir avec discernement afin d'assurer la paix et le salut des hommes sur terre ${ }^{22}$. Le motif de circularité comme image du savoir encyclopédique ${ }^{23}$ se manifeste aussi dans la phrase latine tirée de l'Ecclésiaste $(24,8)$, que nous citons dans sa traduction française: "J'ai parcouru seule la voûte des cieux et me suis promenée dans le fond des abîmes » ${ }^{24}$.

Dans les manuscrits enluminés, la miniature reprend le motif circulaire ${ }^{25}$. Assise sur un trône, la dame est au centre, ses bras étendus forment une croix avec son corps; à ses extrémités, on trouve quatre miroirs. Ainsi, on y lit - à gauche et à droite de ses bras - Lati-tudo, puis au-dessus de sa tête Longitudo et, sous ses pieds Profundum. La dame s'étend dans toutes les directions et c'est à elle que les miroirs renvoient

20. J.-C. Mühlethaler, «Un manifeste poétique de 1463 », p. 92 sq.; George Chastelain, Jean Robertet, Jean de Montferrant, Les Douze Dames de Rhétorique, p. 30.

2I. Cité d'après George Chastelain, Jean Robertet, Jean de Montferrant, Les Douze Dames de Rhétorique, p. 142, v. 15.

22. Ibid., p. 142 sq., v. 41-42.

23. J.-C. Mühlethaler, "Un manifeste poétique de 1463 ", p. 92 sq.; voir aussi K. Straub, "Les Douze Dames de Rhétorique” in Text und Bild, p. 113 et 115.

24. L'inscription en latin dit: Gyrum celi circuivi et in fuctibus maris ambulavi, cité d'après J.-C. Mühlethaler, «La citation éclairante», p. 75.

25. Les références se limitent ici au manuscrit exemplaire de Jean de Montferrant qui se trouve à Cambridge, UL, ms. Nn. III.2, la miniature de Profundité au fol 28v. Consultable sur le site <https://cudl.lib.cam.ac.uk/view/MS-NN-00003-00002>. 
leur reflet du monde. L'iconographie élaborée fait encore allusion à la vertu de la prudence, avec son attribut du miroir, et à la conception du microcosme pour visualiser l'idée du savoir encyclopédique ${ }^{26}$. Dame Profundité perçoit par l'activité intellectuelle; elle constitue le centre où les connaissances universelles se trouvent réunies ${ }^{27}$.

Par contre, la peinture à Lausanne ne correspond pas à la miniature. Ici, la personnification n'est même pas représentée comme une dame noble, mais comme une figure ailée (pl. V). Elle «semble plus angelique que humaine", comme Profundité est décrite dans la première strophe et comparée aux oiseaux, elle a l'«ele plus agile»; en plus, elle "parvole la plaine», soit elle traverse en volant le ciel $^{28}$. Apparemment, l'image reprend avec les ailes le motif le plus concret que les strophes suggèrent ${ }^{29}$. En outre, Profundité combine les savoirs philosophique et théologique qu'elle transmet pour le salut des hommes et en vue d'assurer (d'un point de vue politique) le bien commun. En dotant Profundité d'ailes, la peinture met en évidence sa fonction de médiatrice ${ }^{30}$ : elle fait figure d'ange, de messager envoyé par Dieu.

Multiforme Richesse - notre second exemple - représente les multiples dons de la nature et de Dieu, mais aussi les biens acquis par le travail juvénile, soit l'accumulation des connaissances gardées en mémoire. Les pierres précieuses et les fleurs signifient son trésor de vertus et de valeurs morales ${ }^{31}$. La citation empruntée aux Proverbes $(31,29)$, mais transposée de la seconde à la première personne, affirme la supériorité de Multiforme Richesse $^{32}$ sur les autres femmes vertueuses. Dans la Bible, le verset fait

26. Profundité fait suite à la conception de l'homme comme reflet de l'univers, souvent représentée par l'homme (nu) à bout de bras et inscrit dans le rond du monde. Voir K. Straub, "Les Douze Dames de Rhétorique” in Text und Bild, p. 117-121.

27. J.-C. Mühlethaler, «La citation éclairante», p. 75; George Chastelain, Jean Robertet, Jean de Montferrant, Les Douze Dames de Rhétorique, p. 77.

28. Cité d'après George Chastelain, Jean Robertet, Jean de Montferrant, Les Douze Dames de Rhétorique, p. 142, v. 14, 4, 10.

29. Cf. George Chastelain, Jean Robertet, Jean de Montferrant, Les Douze Dames de Rhétorique, p. 77.

30. J.-C. Mühlethaler, «Un manifeste poétique de $1463 »$, p. 93.

31. Voir K. Straub, "Les Douze Dames de Rhétorique" in Text und Bild, p. 142 sq., avec références.

32. Multe filie congregaverunt divicias, ego supergressa sum universas, cité d'après J.-C. Mühlethaler, "La citation éclairante», p. 77. Cf. George Chastelain, Jean Robertet, Jean de Montferrant, Les Douze Dames de Rhétorique, p. 146, n. 181. 
partie de la louange adressée à la bonne maîtresse de maison dont le prix «surpasse de beaucoup celui des perles» $(31,10)$, comparaison qui pourrait expliquer l'importance accordée aux pierres précieuses dans l'Enseigne: "Perles, saphirs, precieuses jacinctes..." ${ }^{33}$. L'étonnante transformation de la ménagère soucieuse du bon déroulement des affaires en Multiforme Richesse thésaurisant les biens idéels s'inspire peutêtre de la Glossa ordinaria: l'épouse vertueuse y est la figure de la sancta Ecclesia ${ }^{34}$.

Dans la miniature ${ }^{35}$, la personnification est à nouveau présentée trônant et entourée de multiples signes de richesse: sa robe est brodée de perles, ses pieds posés sur un sac dont s'échappent des pièces d'or, dans ses mains, elle tient deux coffrets contenant l'un les «biens de nature» et l'autre les «biens de doctrine». Sa tête dorée et auréolée évoque la gloire qui résulte de son assiduité juvénile. La miniature traduit le contenu des strophes dans son propre langage visuel. L'iconographie s'inscrit dans la continuité des représentations des vertus en se référant par exemple aux modèles de Largesse et de Prudence dont elle récupère le motif du sac aux pièces. Les pierres précieuses et les coffrets, allégories des richesses de l'esprit, renvoient plus précisément au domaine de la rhétorique en visualisant des métaphores propres à signifier l'ornatus dont les tropes (comprenant métaphore et allégorie) sont la manifestation la plus importante ${ }^{36}$.

L'idée d'une fortune multiple et débordante s'exprime sur le panneau peint à Lausanne par la corne d'abondance, symbole de la richesse hérité de l'Antiquité, remplaçant ici le coffre rempli de pierres précieuses et de fleurs ${ }^{37}$, et que la Dame évoque dans la seconde strophe en faisant allusion à ses vertus (pl. VI). La dame est aussi habillée précieusement, elle porte des vêtements ornés, des bijoux et une parure embellie de perles

33. Cité d'après George Chastelain, Jean Robertet, Jean de Montferrant, Les Douze Dames de Rhétorique, p. 146, v. 9.

34. Patrologia Latina CXIII, p. 1114.

35. Cambridge, UL, ms. Nn. III.2, fol. 31v (<https://cudl.lib.cam.ac.uk/view/ MS-NN-00003-00002>).

36. Pour les références et les modèles iconographiques, voir K. Straub, "Les Douze Dames de Rhétorique "in Text und Bild, p. 144-155.

37. George Chastelain, Jean Robertet, Jean de Montferrant, Les Douze Dames de Rhétorique, p. 146, v. 10-11. 
sur la tête, ce qui lui donne, conformément au texte, une apparence "plus que royale» ${ }^{38}$.

Les deux exemples suffisent à illustrer les différences entre les enluminures - qui complètent les strophes en les transformant dans leur propre langage - et les peintures murales. Certes, les strophes ainsi que les citations latines sont peintes sur la paroi, mais les images ne suivent pas l'iconographie élaborée des miniatures. Elles font figure de parents pauvres, car elles sont réduites à une variation des tenues et des vêtements; il semble qu'elles ont été exécutées de manière indépendante, suivant un dessein qui leur est propre.

Ces remarques semblent être également valables pour les représentations des Vertus personnifiées des nobles d'après le Bréviaire des nobles d'Alain Chartier, écrit dans les années 1420-1425. L'œuvre du secrétaire et notaire de Charles VII est en général marquée par la réflexion sur les devoirs de la noblesse en ces temps où sévit la guerre de Cent Ans ${ }^{39}$. Le Bréviaire offre le condensé de l'idéal aristocratique avec sa suite de treize ballades, au fil desquelles s'élabore une sorte de code d'honneur et de vertu pour la noblesse ${ }^{40}$. Il s'ouvre par Noblesse qui se présente comme une princesse accompagnée de douze suivantes: ce sont les Vertus Foy, Loyauté, Honneur, Droicture, Prouesse, Amour, Courtoisie, Diligence, Netteté, Largesse, Sobriété et Persévérance ${ }^{41}$. Les Dames servent de modèles, enseignant aux nobles la façon d'être à nouveau dignes de leur état ${ }^{42}$.

Même si le texte, conservé dans plus de cinquante manuscrits, a été largement diffusé, il ne possède pas de tradition iconographique. Il y a, à ce qu'on sait, un seul manuscrit (Paris, BnF, naf. 18145) qui comporte un cycle d'images représentant les Vertus des nobles, imaginé par Jean Poyer, enlumineur français dans les années 1490-1495 43. C’est le seul

38. Ibid., p. 146 , v. 16.

39. E. J. Hoffman, Alain Chartier, p. 40 sq. et 9-33 pour la biographie de Chartier; cf. Alain Chartier, The Poetical Works, p. 1-27.

40. E. J. Hoffman, Alain Chartier, p. 115.

4I. Pour ce texte, cf. Alain Chartier The Poetical Works, p. 393-409.

42. Sur ce texte et son contexte (la crise de la chevalerie), cf. C. Taylor, «Alain Chartier and Chivalry".

43. Cf. P. Charron, "Une mise en image du discours moral à la fin du Moyen Âge», p. 2. 
point de comparaison possible pour les peintures murales du château Saint-Maire.

Foy, la première compagne de dame Noblesse, décrit la hiérarchie du monde soumis à Dieu qui a assigné une place à chacun dans les trois états de la société. Bien que la peinture ait beaucoup souffert, on distingue encore une femme à la tête voilée, ressemblant à une religieuse (pl. VII). Dans sa main, elle tient le modèle d'une église comme le font les fondatrices dans la tradition iconographique. Par contre, la miniature correspondante montre une dame noble qui porte son regard au ciel où siège la Trinité ${ }^{44}$.

Les représentations de la quatrième Dame, Honneur, se ressemblent dans la mesure où l'on y trouve l'attribut du miroir. Mais il semble que cet attribut soit tout simplement inspiré du texte qui définit Honneur comme "mirouer ou il [s. e. le noble] se doit mirer" ${ }^{45}$ poussé par un amoureux désir. Tandis que dans la miniature ${ }^{46}$, la dame présente seulement l'attribut dans sa main gauche et tient probablement de l'autre la lettre de noblesse, l'idée de l'autoréflexion s'exprimant dans la peinture murale: Dame Honneur, en effet, contemple son reflet dans le miroir. En plus, elle tient un sac rempli de pièces dans l'autre main, ce qui se réfère à la description de l'Honneur comme "trésor de Noblesce, / Son espergne [épargne], sa richesce» ${ }^{47}$.

Pour résumer, force est de constater à travers ces brèves comparaisons que les miniatures ne sont pas à l'origine des peintures murales, ni pour les Douze Dames de Rhétorique ni pour le Bréviaire des nobles. Il semble plutôt que les images du décor peint répondent plus simplement au poème en s'inspirant des attributs que les vers assignent aux dames. Elles résultent d'une visualisation directe de certains mots présents dans les strophes, qu'on a sortis de leur contexte. De cette façon, les personnifications apparaissent sous une forme simplifiée et leurs caractéristiques ne suffisent pas pour les identifier à l'aide de leurs attributs. La composition semble due à la demande de reproduire sur

44. Paris, BnF, naf. 18145, fol. 93. Consultables sur le site <http://images.bnf.fr/jsp/ index.jsp>.

45. Vers cité d'après Alain Chartier The Poetical Works, p. 397, v. 87. Concernant la miniature, voir P. Charron, "Une mise en image du discours moral à la fin du Moyen Âge", p. 9.

46. Paris, BnF, naf. 18145, fol. 95.

47. Cité d'après Alain Chartier, The Poetical Works, p. 397, v. 85 sq. 
les parois le texte des deux poèmes dont les strophes occupent l'espace principal; les représentations en dépendent.

La grande importance attachée au texte soulève la question de l'intention d'Aymon de Montfalcon, qui a fait décorer le corridor de ces deux poèmes, et de la manière dont il a pu en avoir connaissance. Le Bréviaire des nobles était très répandu jusqu'au $\mathrm{XVI}^{\mathrm{e}}$ siècle et son auteur fut admiré comme "le pere de l'eloquence françoyse " ${ }^{48}$. Évidemment, Aymon de Montfalcon comptait aussi parmi ses nombreux admirateurs. Fin lettré, il a composé lui-même des poésies avant d'être nommé princeévêque; on lui attribue plusieurs poèmes influencés par l'œuvre d'Alain Chartier ${ }^{49}$. Le choix d'un de ses textes comme décor du palais épiscopal pourrait donc s'expliquer par l'admiration générale dont jouissait ce poète français même en dehors du royaume ${ }^{50}$.

Quant au traité des Douze Dames de Rhétorique, plusieurs voies de transmission des Pays-Bas bourguignons à Lausanne semblent possibles. Il y a tout d'abord un lien de parenté: Aymon de Montfalcon appartenait à une famille noble du Bugey ${ }^{51}$ et l'une de ses sœurs était mariée à un fils de la famille de Montferrand. Ainsi, Aymon était apparenté à Benoît de Montferrand, son prédécesseur au poste d'évêque de Lausanne et dont le frère n'était autre que ce Jean de Montferrant ${ }^{52}$ qui avait participé à la

48. C'est ainsi que le présente Pierre Fabri dans son Grand et vray art de pleine rhétorique (1521) : voir F. Bouchet, «A Good Carter as Guide», p. 325. On connaît plus de cinquante manuscrits du XVe et XVI $\mathrm{XV}^{\mathrm{e}}$ siècle qui contiennent le Bréviaire des nobles: voir O. Robinson, "Alain Chartier: The Manuscript and Print Tradition», p. 247-250.

49. On lui attribue la Pastourelle et le Procès du banny a jamais du Jardin d'amours contre la volonté de sa dame dont il n'existe qu'une transcription moderne par extraits. Aymon a probablement aussi composé un autre poème intitulé Le Débat du Gris et $d u$ Noir (conservé dans deux manuscrits, Paris, BnF, ms fr. 25421 et ms Rothschild 2798); Cf. Antitus, Poésies, p. X sq.

50. Voir E. Cayley, A. Kinch (dir.), Chartier in Europe.

5I. M. Reymond, "Aymon de Montfalcon, évêque de Lausanne, 1491-1517», p. 31.

52. C. Chavannes-Mazel, qui a réussi à identifier Jean de Montferrant comme coauteur et commanditaire du manuscrit de Cambridge, a proposé cette voie de transmission du texte à Lausanne ("The Twelve Ladies of Rhetoric in Cambridge (CUL MS Nn.3.2)», p. 140 sq. et 149 sq.). Une représentation de l'évêque Benoît de Montferrand et de son blason est conservée au château Saint-Maire, voir M. Grandjean, La ville de Lausanne, p. 365. 
correspondance des Douze Dames de Rhétorique, commandant aussi le plus ancien manuscrit enluminé 53 .

Il serait également envisageable que Montfalcon ait eu connaissance des Douze Dames au cours des négociations qui se déroulèrent à Bruxelles autour du mariage du duc Philibert le Beau (1480-1504) avec Marguerite d'Autriche (1480-1530), fille de Maximilien Ir et de Marie de Bourgogne. Aymon de Montfalcon avait été chargé de cette mission et c'est lui qui célébra le mariage par procuration en $1501^{54}$. Même si Chastelain, l'auteur des "Enseignes" des Douze Dames, était déjà mort depuis quelque vingt-cinq ans, il était considéré comme un maître par les poètes franco-bourguignons. Parmi ces admirateurs se trouvait Jean Lemaire de Belges qui a été choisi plus tard par Marguerite d'Autriche pour superviser l'édification du monastère de Brou à Bourg-en-Bresse ${ }^{55}$. Ce chef-d'œuvre de l'art gothique flamand a été conçu en mémoire de Philibert (mort en 1504) et fut destiné à abriter son tombeau ainsi que ceux de sa mère et de Marguerite.

Il serait également possible que le poète Antitus Favre ait attiré son attention sur les Douze Dames. À partir de 1499 environ, il était au service d'Aymon en qualité de chapelain. Le poète franc-comtois compte parmi les savants et les hommes de lettres dont le prince-évêque s'entourait ${ }^{56}$. Il semble qu'Antitus était familier de la littérature francobourguignonne ${ }^{57}$, ce que laisse entrevoir le recueil de ses poèmes (conservés aux Archives cantonales vaudoises) contenant Le Portail du temple Bocace qui se présente comme une continuation du Temple de Bocace de George Chastelain, l'auteur des «Enseignes» des Douze

53. Cf. supra, p. 134.

54. Aymon était en relations étroites avec la maison ducale de Savoie pour laquelle il accomplit de nombreuses missions diplomatiques; voir Helvetia sacra, I/4, p. 146. Au temps des négociations de mariage, il commanda une tapisserie à Bruxelles; voir M. Reymond, "Aymon de Montfalcon, évêque de Lausanne, 1491-1517 », p. 99 et 107. Pendant la régence de Marguerite d'Autriche, gouvernante des Pays-Bas, l'évêque fit fabriquer - toujours à Bruxelles - des parements liturgiques, actuellement conservés au Bernisches Historisches Museum; cf. A. Stauffer, D'or et de soie ou les voies du salut.

55. Voir R. Meyenberg, Alain Chartier prosateur et l'art de la parole au XVe siècle, p. 38; M. Hörsch, Architektur unter Margarete von Österreich, Regentin der Niederlande (1507-1530).

56. Antitus, Poésies, p. XI.

57. É. Bousmar, «Faut-il restituer Antitus Favre à la littérature bourguignonne?». 
Dames de Rhétorique ${ }^{58}$. En outre, un de ses écrits porte la dédicace: "A tres reverend pere en Dieu, Amé de Montfalcon, evesque et prince de Lausanne $[\ldots]$ » qui "desirez veoir gracieuses inventions poethiques» 59 . Il confirme enfin le goût de l'évêque pour la poésie en parlant de "celluy qui ayme rethorique» ${ }^{60}$.

Cet intérêt littéraire, semble-t-il, se reflète bien dans le sujet choisi par le prince-évêque pour le décor de sa résidence. À cette époque, les thèmes allégoriques étaient "à la mode», sous forme de tapisseries ou de peintures murales. Les images des Vertus, mais aussi des cycles de personnages historiques, ornaient les palais princiers. Ils servaient à mettre en scène leur pouvoir en représentant les modèles dont on prétendait s'inspirer.

Un des exemples les plus connus est les Neuf Preux. Un texte du $\mathrm{XIV}^{\mathrm{e}}$ siècle, les Voeux du paon de Jacques de Longuyon, est à la source de l'ensemble de neuf héros qui incarnent toutes les vertus du parfait chevalier. Il s'agit de personnages issus d'une lignée royale: trois héros de l'Antiquité (Hector, Alexandre le Grand, Jules César) y côtoient trois héros de l'Ancien Testament (Josué, le roi David, Judas Maccabée) et des héros chrétiens, Arthur, Charlemagne et Godefroi de Bouillon. Ils représentent souvent le bon gouvernement: ainsi, on retrouve les Neuf Preux comme décor des mairies aussi bien que des demeures aristocratiques comme le château de La Manta à Saluces, dont les fresques de la sala baronale furent réalisées dans les années 1410-1430 ${ }^{61}$.

Ces personnages fameux étaient faciles à identifier, de même que des représentations des prophètes ou d'allégories comme les arts libéraux ou les vertus cardinales, car ils possédaient une propre tradition iconographique. Contrairement à ces ensembles, les Vertus des nobles et les Douze Dames de Rhétorique n'étaient pas reconnaissables grâce aux seules images; les strophes explicatives étaient nécessaires. Mais le fait que le texte occupe tellement d'espace est déjà - semble-t-il - une

58. Antitus, Poésies, p. XXV. Sur le recueil, cf. aussi la contribution d'E. Doudet dans ce volume.

59. La Satyre Megere, citée d'après Antitus, Poésies, I, v. 1-6, p. 3.

6o. Les Quatre Eages passees, cités d'après Antitus, Poésies, II, v. 332, p. 41.

6I. Sur les Neuf Preux et les peintures à la Manta, voir I. Rauch, D. Täube, H. Westermann-Angerhausen, Die gute Regierung, p. 11, 18-21 et 23; L. Rochat, Les peintures du corridor du château Saint-Maire à Lausanne, p. 72; S. Roettgen, Wandmalerei der Frührenaissance in Italien, p. 42-59, 447, 451 et 454, avec références. 
sorte de déclaration en faveur de la suprématie de l'écrit. De surcroît, l'ensemble à Saint-Maire paraît être plus particulièrement lié à la personne du commanditaire. Les deux cycles servent à exprimer un idéal au moyen de douze personnifications. Le nombre est connoté positivement, car il rappelle le nombre des apôtres (mais aussi des petits prophètes, des sibylles et des pairs de Charlemagne), un groupe élu appelé à propager l'Évangile ${ }^{62}$.

L'ensemble suit une structure circulaire. En entrant dans le corridor, le sens de la lecture commence à gauche par la première des douze Dames. Les Dames figurent non seulement l'idéal du poète qui est un créateur savant, guidé par la morale et inspiré par Dieu; les poèmes manifestent aussi l'importance de la rhétorique dans le domaine politique en faisant écho aux orateurs de l'Antiquité - Cicéron et Quintilien - qui mettaient leur art au service du bien commun. Les Douze Dames s'adressent ainsi aux personnes responsables du bien-être du peuple, c'est-à-dire aux souverains et à leurs conseillers. Pour mettre en valeur la rhétorique, le texte fait aussi référence aux qualités du prince parfait décrit dans les Miroirs des princes, des manuels qui définissaient le bon gouvernement ${ }^{63}$.

À la fin du cycle des Douze Dames, on découvre un treizième panneau. C'est qu'à la différence des Vertus des nobles qui accompagnent Dame Noblesse, les Dames de Rhétorique se composent de seulement douze personnages, de sorte qu'il a fallu compléter la série par un dernier panneau sur la paroi nord. Ce treizième panneau énigmatique diffère visiblement des autres en présentant un couple qui tient un grand écusson, entouré des banderoles (pl. VIII.1). Le couple peut être identifié par les noms inscrits à côté de leurs têtes: il s'agit d'Adam et Ève. Une identification étonnante puisque suivant leur iconographie courante, le premier couple est présenté soit nu, soit au travail après la chute. Cependant, ici, ils sont habillés à la façon de personnages dignes: Adam, à la barbe et aux cheveux longs, porte une robe boutonnée et coûteuse. Ėve est vêtue d'une robe à larges manches, elle porte un collier de perles et sur sa tête une sorte de turban aux bandes de textile qui retombent sur les épaules.

Néanmoins, les inscriptions semblent soutenir leur identification: elles indiquent sous la figure d'Adam "Mestres, clercs, villains et gentilz / Sont de nous deux filles et filz» et sous la figure d'Ève «Notre naissance

62. Voir J.-C. Mühlethaler, "Un manifeste poétique de 1463 », p. 86.

63. Voir K. Straub, "Les Douze Dames de Rhétorique" in Text und Bild, p. 256-261. 
retenez / et l'ung l'aultre en amour tenez", c'est-à-dire "souvenez-vous de notre naissance / et aimez-vous l'un l'autre» ${ }^{64}$.

Sur l'écusson sont figurés, de haut en bas: le Pape avec la grosse clé entouré d'ecclésiastiques, l'Empereur l'épée en main et au centre, des personnages de la cour, en dessous un paysan qui conduit une charrue traînée par deux chevaux et enfin un bûcheron brandissant sa cognée ${ }^{65}$. L'inscription au-dessus de l'écusson précise: "Cet escu fait signifiance/ Que nous fûmes d'une naissance / Et par sagement maintenir / Peut petit homme hault venir", et l'inscription au-dessus du paysan précise, qu'il n'est pas méprisé par les Papes ou les rois puisque tous les hommes sont issus du même moule ${ }^{66}$. L'énoncé se lit comme une déclaration de l'égalité de tous les hommes devant Dieu ${ }^{67}$ indépendamment de leur statut social. Bien évidemment, l'égalité est liée à une bonne conduite de vie selon les normes de la sagesse et de la morale chrétienne, mais dans un cadre strict d'une société bien définie par l'ordre des trois états telle que la présente l'écusson ${ }^{68}$. C'est l'espoir du salut, soutenu par Adam et Ėve, les parents du genre humain. En évoquant la chute, ils rappellent aux hommes qu'ils sont pécheurs et mortels.

$\mathrm{Au}$ travers des inscriptions, le dernier panneau invite à un mode de vie chrétienne. Enfin, l'écusson présente le souverain ecclésiastique et le régent séculier à qui le prince-évêque était subordonné. En rassemblant les états de la société féodale, il renvoie en même temps aux fonctions d'Aymon de Montfalcon, lui qui appartient comme prince-évêque aussi bien au clergé qu'à la noblesse et est en charge du bien du peuple. Il semble donc logique que le tour dans le corridor continue, sur l'autre paroi, avec Dame Noblesse suivie de son groupe d'allégories.

Pour conclure, les peintures murales apparaissent bien comme un ensemble d'un temps de transition: les ornements, le décor pictural et la

64. Cité d'après J.-A. Bohy, «Les fresques pré-renaissantes du château de Lausanne», p. 134.

65. Ibid., p. 134.

66. «Papes, Roys ne me desgettez / Car d'ung mole [moule] fumes gettez» (Ibid.).

67. Ibid.

68. Cf. A. Taverney, «Les peintures et les inscriptions du corridor du château de Lausanne», p. 14 sq. Partant de l'écusson, A. Taverney interprète les personnifications sur la paroi nord, soit les Douze Dames, avant leur identification par M.-R. Jung, comme des vertus bourgeoises. 
devise tirée de Virgile les orientent vers la Renaissance. Elles représentent le goût personnel et l'admiration du commanditaire pour les textes d'Alain Chartier et de George Chastelain autant que ses fonctions, même si la référence à son office ecclésiastique se limite au treizième panneau. Avant tout, l'ensemble met la noblesse en relation avec la rhétorique ${ }^{69}$. Même si elles sont détachées de leur contexte d'origine, les Douze Dames exposent des qualités morales et éthiques, le savoir et des connaissances universelles qu'on attend de l'élite aristocratique. Elles présentent un modèle, lequel inclut l'éloquence (la rhétorique) : s'y reflète la conception du prince parfait issue de la tradition médiévale des Miroirs des princes.

Par leurs attributs, les personnifications s'inscrivent dans le sillage du modèle traditionnel des Vertus et des héros exemplaires. Ainsi, les peintures murales se rattachent par leur programme d'images aussi bien que par leur format aux représentations princières sous forme de tapisseries, fresques ou tableaux vivants qui servent à célébrer la vie idéale de cour. Mais, en fin de compte, il s'agit d'un cycle d'images nouvelles, inventé uniquement dans le but de décorer la résidence du princeévêque de Lausanne. En commandant cet ensemble singulier, Aymon de Montfalcon se présente lui-même comme une sorte de créateur.

Karen Straub Conservatrice au Museum Schnütgen de Cologne

69. Voir M.-R. Jung, "Les “Douze Dames de Rhétorique” ", p. 231. 


\section{BIBLIOGRAPHIE}

\section{Sources}

Alain Chartier, The Poetical Works of Alain Chartier, ed. by James C. Laidlaw, Cambridge, Cambridge University Press, 1974.

Antitus, Poésies, éd. par Manuela Python, Genève, Droz, 1992 (Textes littéraires français, 422) [éd. du mss. ACV, P Antitus, numérisé sous <http://www.e-codices.unifr.ch/en/list/one/acv/P-Antitus $>$ ].

George Chastelain, Jean Robertet, Jean de Montferrant, Les Douze Dames de Rhétorique, éd. par David Cowling, Genève, Droz, 2002.

\section{Travaux}

Boнy, Juliette-Alice, «Les fresques pré-renaissantes du château de Lausanne", Indicateur d'antiquités suisses, 40/2 (1938), p. 129-141. —, "Les peintures du château de Lausanne. Première œuvre renaissante en Suisse", Revue historique vaudoise, 47/2 (1939), p. 57-80.

Bouchet, Florence, "A Good Carter as Guide: Imitating Alain Chartier", in A Companion to Alain Chartier (c. 1385-1430). Father of French Eloquence, ed. by Daisy Delogu, Joan E. MacRae, Emma Cayley, Leiden/Boston, Brill, 2015, p. 324-353.

Bousmar, Éric, "Faut-il restituer Antitus Favre à la littérature bourguignonne? Connections austro-bourguignonnes dans la carrière et l'œuvre d'un rhétoriqueur peu connu ( $\dagger$ entre 1501 et 1506)", in "A l'heure encore de mon escrire». Aspects de la littérature de Bourgogne sous Philippe le Bon et Charles le Téméraire, éd. par Claude Thiry, Louvain-la-Neuve, Les Lettres Romanes, 1997, p. 9-22.

Cayley, Emma, Kinch, Ashby (dir.), Chartier in Europe, Cambridge, Brewer, 2008. 
Charron, Pascale, "Une mise en image du discours moral à la fin du Moyen Âge: le cas du manuscrit du Secret des secrets et du Bréviaire des nobles enluminé par Jean Poyer (Paris, BnF, ms. n. a. fr. 18145)", in Quand l'image relit le texte; regards croisés sur les manuscrits médiévaux, éd. par Sandrine Heriché-Pradeau, Maud Pérez-Simon, Paris, Presses Sorbonne Nouvelle, 2013, p. 221-232 et en ligne <https://halshs.archives-ouvertes.fr/halshs-00948483>.

Chavannes-Mazel, Claudine, "The Twelve Ladies of Rhetoric in Cambridge (CUL MS Nn.3.2)", Transactions of the Cambridge Bibliographical Society, X/2 (1992), p. 139-155, fig. 5-12.

Delmarcel, Guy, Los Honores. Tapisseries flamandes pour Charles Quint, Anvers, Pandora/Snoeck-Ducaju, 2000.

Doudet, Estelle, "Poétique en mouvement: Le beau "débat" des Douze Dames de Rhétorique", in Poétiques en transition: entre Moyen Âge et Renaissance, éd. par Jean-Claude Mühlethaler, Jacqueline Cerquiglini-Toulet, Études de Lettres, 263 (2002/4), p. 83-110.

Elsig, Frédéric, "La peinture en Savoie et en Franche-Comté durant la première moitié du XVI ${ }^{\mathrm{e}}$ siècle", in La Renaissance en Savoie. Les arts au temps du duc Charles II (1504-1553), éd. par Mauro Natale, Frédéric Elsig, Genève, Musée d’Art et d'Histoire, 2002, p. 77-94.

Grandjean, Marcel, La ville de Lausanne. Introduction, extension urbaine, ponts, fontaines, édifices religieux (sans la cathédrale), hospitaliers, édifices publics, Bâle, Birkhaüser, 1965 (Les Monuments d'art et d'histoire du canton de Vaud, 1).

Helvetia Sacra, I/4. Le diocèse de Lausanne (VI siècle-1821), de Lausanne et Genève (1821-1925) et de Lausanne, Genève et Fribourg (depuis 1925), par un groupe d'auteurs, rédaction Patrick Braun, Bâle/ Francfort-sur-le-Main, Helbing \& Liechtenhahn, 1988.

Hoffman, Edward Joseph, Alain Chartier. His Work and Reputation, Genève, Slatkine Reprints, 1975 (1942).

Hörsch, Markus, Architektur unter Margarete von Österreich, Regentin der Niederlande (1507-1530). Eine bau- und architekturgeschichtliche Studie zum Grabkloster St. Nicolas-deTolentin in Brou bei Bourg-en-Bresse, Brüssel, Palais der Academie, 1994 (Verhandelingen van de Koninklijke Academie voor Wetenschappen, Letteren en Schone Kunsten von België, Klasse der Schone Kunsten, 58). 
Jodogne, Pierre, "Les "Rhétoriqueurs" et l'humanisme", in Humanism in France at the End of the Middle Ages and in the Early Renaissance. Papers presented at a Symposium held by the School of French of the University of Warwick, April 1969, ed. by Anthony H. T. Levi, Manchester, Manchester University Press, 1970, p. 150-175 (Tübinger Beiträge zur Linguistik, 175).

Jung, Marc-René, "Les "Douze Dames de Rhétorique" ", in Du mot au texte. Actes du III colloque international sur le moyen français, éd. par Peter Wunderli, Tübingen, Narr, 1982, p. 229-240.

Meyenberg, Regula, Alain Chartier prosateur et l'art de la parole au $X V^{e}$ siècle. Études littéraires et rhétoriques, Bern, Francke, 1992.

Mühlethaler, Jean-Claude, "Un manifeste poétique de 1463: les "Enseignes" des Douze Dames de Rhétorique», in Les grands Rhétoriqueurs. Actes du $V^{e}$ colloque international sur le moyen français, t. I, Milan, Vita e pensiero, 1985, p. 83-101.

—, "La citation éclairante: Les épigraphes dans les "Enseignes" des Douze Dames de Rhétorique", in Poétiques en transition: entre Moyen Âge et Renaissance, éd. par Jean-Claude Mühlethaler, Jacqueline Cerquiglini-Toulet, Études de Lettres, 263 (2002/4), p. 73-81.

Piaget, Arthur, "Aymon de Montfaucon et sa cour littéraire», in Mélanges de linguistique et de littérature offerts à $M$. Alfred Jeanroy par ses élèves et ses amis, Paris, Droz, 1928, p. 447-467.

Rauch, Ivo, Täube, Dagmar, Westermann-Angerhausen, Hiltrud, Die gute Regierung. Vorbilder der Politik im Mittelalter, Köln, Katalog zur Ausstellung im Schnütgen-Museum Köln, 2001.

Reymond, Maxime, "Les châteaux épiscopaux, les hôtels de ville de Lausanne", in Mémoires et documents publiés par la Société d'histoire de la Suisse romande, 2e série/IX, 1911, p. 110-228 et 232-253.

—, "Aymon de Montfalcon, évêque de Lausanne, 1491-1517», Revue d'histoire ecclésiastique suisse, 14 (1920), p. 28-39 et 99-111.

Robinson, Olivia, "Alain Chartier: The Manuscript and Print Tradition", in A Companion to Alain Chartier (c. 1385-1430). Father of French Eloquence, ed. by Daisy Delogu, Joan E. MacRae, Emma Cayley, Leiden/Boston, Brill, 2015, p. 223-252. 
Rochat, Lise, Les peintures du corridor du château Saint-Maire à Lausanne, Mémoire d'histoire de l'art moderne, sous la direction de Prof. Mauro Natale, Université de Genève, 2005 (inédit).

Roettgen, Steff, Wandmalerei der Frührenaissance in Italien, Bd. I: Anfänge und Entfaltung 1400-1470, München, Hirmer, 1996.

Stauffer, Annemarie, D'or et de soie ou les voies du salut. Les ornements sacerdotaux d'Aymon de Montfalcon, évêque de Lausanne, catalogue d'exposition, Berne, Musée d'histoire, 2001.

Straub, Karen, " "Se riens y a qui de noble umbrage, / Qui soit de fruit ou de cler fenestrage / Pour decorer roayal palais ou porge / Soit pris en gré" ", in Poètes et artistes. La figure du créateur en Europe au Moyen Âge et à la Renaissance, éd. par Sophie Cassagnes-Brouquet, Martine Yvernault, Limoges, Presses universitaires de Limoges, 2007, p. 273-288.

—, "Les Douze Dames de Rhétorique" in Text und Bild. Allegorisches Manifest und literarische Debatte an den Höfen von Burgund und Bourbon, Affalterbach, Didymos-Verlag, 2016 (Studien zur Kunstgeschichte des Mittelalters und der Frühen Neuzeit, 15).

Taverney, Adrien, "Les peintures et les inscriptions du corridor du château de Lausanne", Association du Vieux-Lausanne (Rapport du Comité sur la gestion pendant l'année 1929), Lausanne, 1930, p. 12-16.

TAYLOR, Craig, "Alain Chartier and Chivalry: Debating Knighthood in the Context of the Hundred Years War", in A Companion to Alain Chartier (c. 1385-1430). Father of French Eloquence, ed. by Daisy Delogu, Joan E. MacRae, Emma Cayley, Leiden/Boston, Brill, 2015, p. 141-162.

Zumthor, Paul, Le masque et la lumière. La poétique des grands rhétoriqueurs, Paris, Éditions du Seuil, 1978. 